\title{
Maternal and Neonatal Outcomes after Attempted External Cephalic Version among Women with One Previous Cesarean Delivery
}

\author{
Rodney A. McLaren Jr., MD ${ }^{1}$ Fouad Atallah, MD ${ }^{1} \quad$ Nelli Fisher, MD ${ }^{1}$ Howard Minkoff, MD ${ }^{1}$ \\ ${ }^{1}$ Department of Obstetrics and Gynecology, Maimonides Medical \\ Center, Brooklyn, New York \\ Address for correspondence Rodney A. McLaren Jr., MD, Department \\ of Obstetrics and Gynecology, Maimonides Medical Center, 967, \\ 48th Street, Brooklyn, NY 11219 (e-mail: rmclaren624@gmail.com). \\ Am J Perinatol Rep 2018;8:e349-e354.
}

\begin{abstract}
Objective This study was aimed to evaluate success rates of (1) external cephalic version (ECV) among women with one prior cesarean delivery $(C D)$ and $(2)$ maternal and neonatal outcomes after ECV among women with prior CD.

Study Design Two linked studies using U.S. Natality Database were performed. First we performed a retrospective cohort comparing ECV success rates of women with prior $C D$ and women without prior CD. Then we compared the outcomes of TOLACs (trial of labor after cesarean delivery) that occurred after ECV with those that occurred without ECV. Multivariable logistic regression analysis was used to estimate adverse outcomes. Results A total of 715 women had ECV after 36 weeks with prior CD and 9,976 had ECV without prior scar. ECV success rate with scar was $80.6 \%$ and without scar was

Keywords

- blood transfusion

- cesarean delivery

- external cephalic version

- trial of labor after cesarean

- unplanned hysterectomy 86.4\% $(p<0.001)$. Seven hundred and sixteen women underwent TOLAC after ECV attempt and 234,617 underwent TOLAC without a preceding attempt. Women with preceding version had increased risks of maternal transfusion ( 1 vs. $0.4 \%$, adjusted OR [odds ratio]: 2.48 [ $95 \% \mathrm{Cl}$ (confidence interval): 1.17-5.23]), unplanned hysterectomy (0.4 vs. $0.06 \%$, adjusted OR: 6.90 [95\% Cl: 2.19-21.78]), and low 5-minute Apgar's score (2.5 vs. $1.5 \%$, adjusted OR: 1.76 [95\% Cl: $1.10-2.82]$ ).

Conclusion Women with prior CD may have a decrease in the rate of successful ECV. While the absolute risks are low, ECV appears to increase risks of adverse maternal and neonatal outcomes among women undergoing a trial of labor.
\end{abstract}

Breech presentation at term occurs in 3 to $4 \%$ of all pregnancies. ${ }^{1}$ The three main options of management for women with breech presentation at term are planned vaginal delivery, elective cesarean delivery, and external cephalic version (ECV). Since the results of the Term Breech Trial in 2,000 cesarean delivery (CD) for breech presentation has increased, ${ }^{2}$ putting more emphasis on the option of version for those who want to avoid a CD. ECV is a procedure that involves applying pressure to the woman's abdomen to turn the fetus from breech to vertex with the goal to increase the chances of a vaginal delivery. ${ }^{3}$ Given a

received

September 4, 2018 accepted after revision October 16, 2018
DOI https://doi.org/

10.1055/s-0038-1676297. ISSN 2157-6998. low risk of complication and a decreased risk of $\mathrm{CD}$ among ECV attempts in a meta-analysis, ${ }^{4}$ the American College of Obstetricians and Gynecologists recommends that all women near term with breech presentations should be offered an ECV if they do not have a contraindication for trial of labor. ${ }^{3}$

The success and safety of ECV among women with one previous $C D$ have been reported previously. Multiple studies have shown that the success rate of ECV among women with one previous $C D$ is similar to women without a history of cesarean with a pooled success rate of $73.6 \%$ in 398 women
Copyright $\odot 2018$ by Thieme Medical Publishers, Inc., 333 Seventh Avenue, New York, NY 10001, USA. Tel: +1(212) 584-4662.
License terms

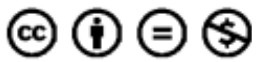


with prior cesarean deliveries. ${ }^{5-12}$ In addition, they reported low complication rates without serious adverse maternal outcomes. However, none of these studies had enough power to detect rare but serious maternal and neonatal outcomes, such as uterine rupture which has a reported prevalence between $0.3-0.7 \%$ among women undergoing trial of labor with history of $\mathrm{CD} .^{13,14}$ There were two goals in this study. One goal was to evaluate the success rate of ECV in women with one prior $\mathrm{CD}$. The second goal was to evaluate maternal and neonatal outcomes after ECV in women with one prior $\mathrm{CD}$ undergoing trial of labor.

\section{Materials and Methods}

We performed two linked studies using the U.S. Natality Database from 2014 to 2016 based on U.S. birth certificates. Study 1 was comprised of women who underwent ECV. Women with one prior $C D$ constituted the study group, and women without a prior CD constituted the control group. Study 2 was comprised of women undergoing trials of labor after cesarean delivery (TOLAC). Women with a prior ECV constituted the study group, while women without a prior ECV constituted the control group. Data were obtained from the National Center for Health Statistics, Centers for Disease Control and Prevention. This dataset is publicly available and contains deidentified data; institutional review board approval was not required.

Analysis was limited to singleton, nonanomalous pregnancies among women who underwent ECV and who underwent a TOLAC. We excluded patients who delivered at less than 36 weeks of gestation, and those who had more than one prior $\mathrm{CD}$.

After the study and control groups were established, the data were reviewed to record maternal age, prepregnancy body mass index (BMI), gestational age at delivery, birth weight, fetal presentation at birth, and mode of delivery. Adverse maternal outcomes included blood transfusion, uterine rupture, postpartum hysterectomy, and intensive care unit admission. Adverse neonatal outcomes included 5-minute Apgar's scores less than 7, immediate assisted ventilation, assisted ventilation greater than 6 hours, neonatal seizures, and neonatal intensive care unit admission.

Sample size calculation was based on a reported prevalence of uterine rupture risk of $0.7 \%$ among trial of labors with an unscarred uterus. ${ }^{13}$ Assuming a prevalence of uterine rupture risk of $4 \%$ in the study group and $0.7 \%$ in the control group, a minimum of 702 patients per group was needed with an $\alpha$ error of 0.005 and $\beta$ error of 0.10 . Assuming a prevalence of $2 \%$ of TOLAC among all births, a reported prevalence of $3 \%$ of breech presentation at term, ${ }^{1}$ a reported prevalence of $25 \%$ not offered $\mathrm{ECV},{ }^{15}$ and $30 \%$ declining $\mathrm{ECV},{ }^{16}$ a total of $2,228,571$ births would need to be reviewed. Assuming the average number of births per year in the U.S. is 4 million, a total of one year of national data was needed. However, in 2016, there were only 279 attempted ECVs after exclusion criteria were applied. Therefore additional 2 years of national data was added to meet the minimum number of subjects required.
Maternal and labor characteristics were compared between the groups in both analyses. Student $t$-test was used for continuous variables and Chi-square test was used with categorical variables. Statistical significance was defined as $p<0.005$. Among women who underwent TOLAC, multivariable logistic regression analyses were performed to predict adverse maternal and neonatal outcomes controlling for potential confounding variables. The main exposure of risk was a prior ECV. Adverse maternal outcomes included any blood transfusion, uterine rupture, unplanned hysterectomy, and intensive care unit admission. The potential confounding variables used for maternal outcomes included maternal age, maternal BMI, and CD. Adverse neonatal outcomes included 5-minute Apgar's score less than 7, immediate need of assisted ventilation, assisted ventilation for more than 6 hours, neonatal seizures, and neonatal intensive care unit admission. The potential confounding variables used for neonatal outcomes included maternal BMI, gestational age at delivery, and birth weight. All analyses were performed on StataCorp LLC Stata 15.1 (College Station, Texas).

\section{Results}

There were 155,332 multiparous women who underwent an ECV identified among 11,943,020 births (1.3\%) between 2014 and 2016. After exclusions, the final cohort for study 1 included 10,691 women (-Fig. 1). There were 715 women with one previous $C D$ and 9,976 women without a prior $C D$. Maternal and obstetrical characteristics were similar between groups ( $\mathbf{- T a b l e ~} \mathbf{1}$ ).

A univariate, unadjusted comparison of success rates and mode of delivery of women with one prior cesarean and without prior cesarean was performed (-Table 2). The success rate of ECV was higher in women without CD compared with women with a prior cesarean (86.4 and $80.6 \%$, respectively; $p<0.001$ ).

For study 2, after exclusions, 235,463 women undergoing trial of labor after one cesarean constituted the final cohort (-Fig.2). There were 716 women undergoing a TOLAC with a preceding version and 234,617 women without a preceding version. Maternal and obstetrical characteristics were similar (-Table 3).

We performed an unadjusted comparison of mode of delivery of women undergoing TOLAC with a preceding version and those without a version (-Table 4). Mode of delivery was similar between groups (vaginal, $p=0.096$; operative, $p=0.042$; cesarean, $p=0.494$ ). Vaginal birth after cesarean occurred in $74.9 \%$ of women undergoing TOLAC after a preceding version, and $73.6 \%$ in women without a preceding version $(p=0.494)$.

Finally, we performed an adjusted comparison of the maternal and neonatal outcomes of the study and control groups (-Table 5). There was no report of a uterine rupture among the women undergoing TOLAC after a preceding version. However, they had an increased risk of maternal blood transfusion (adjusted OR [odds ratio]: 2.48 [95\% CI (confidence interval): 1.17-5.23]), unplanned hysterectomy (adjusted OR: 6.90 [95\% CI: 2.19-21.78]), and 5-minute Apgar's score of 7 (adjusted OR: 1.76 [95\% CI: 1.10-2.82]). 


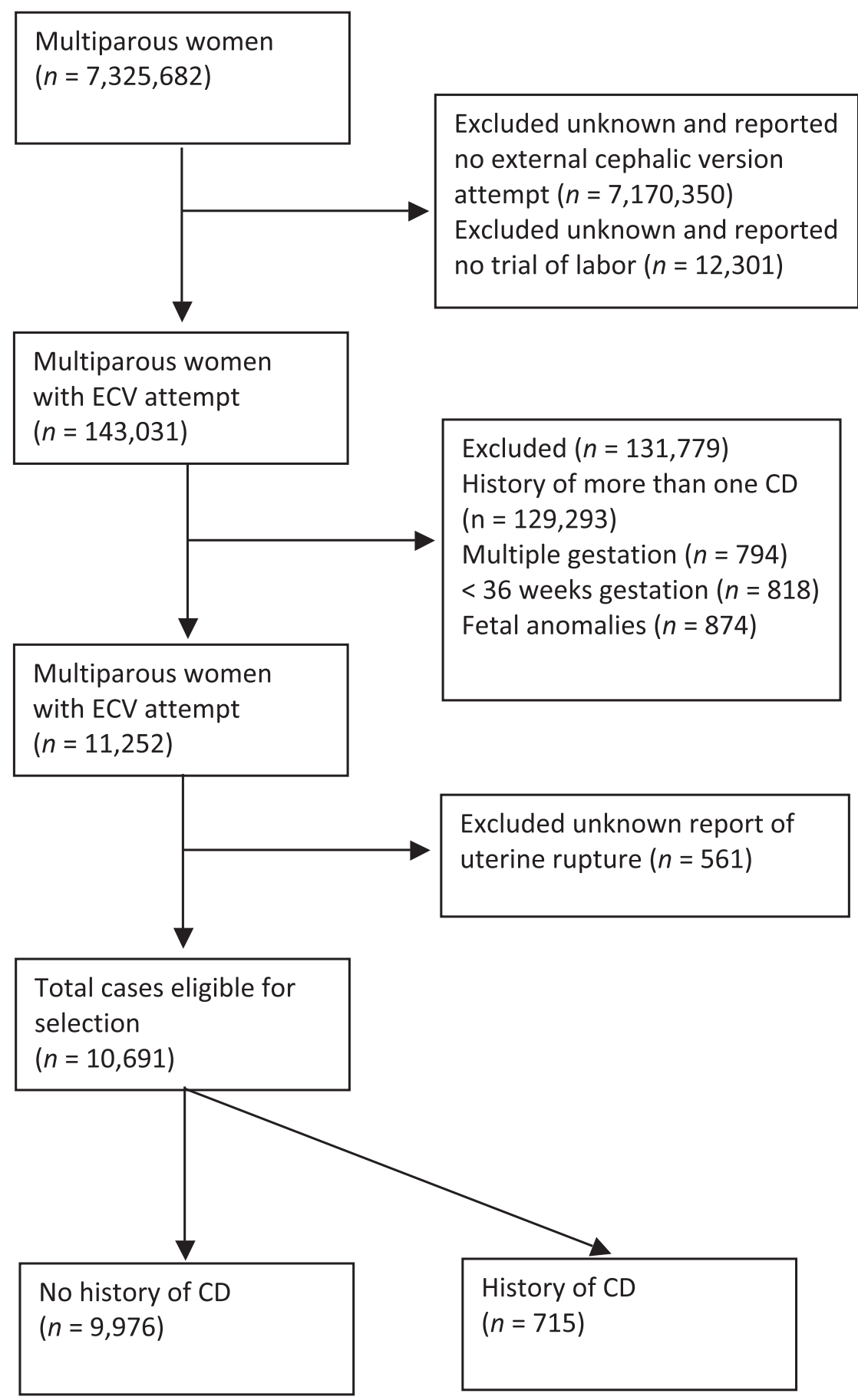

Fig. 1 Cohort selection flow diagram of multiparous women undergoing ECV attempt, study 1. CD, cesarean delivery; ECV, external cephalic version.

Table 1 Maternal and obstetrical characteristics among women with an external cephalic version attempt

\begin{tabular}{|l|l|l|l|}
\hline Characteristic & No history of cesarean delivery & History of cesarean delivery & $p$-Value \\
\hline$n$ & 9,976 & 715 & \\
\hline Maternal age $(\mathrm{y})$ & $30.6+/-5.5$ & $30.7+1-5.2$ & 0.474 \\
\hline BMI & $26.4+/-6.2(9,707)$ & $26.8+/-6.2(704)$ & 0.052 \\
\hline Gestational age (wk) & $39.2+/-1.6(9,971)$ & $39.3+/-1.6$ & 0.051 \\
\hline Birth weight $(\mathrm{g})$ & $3,407.5+/-478.1(9,974)$ & $3,436.0+/-487.8(714)$ & 0.124 \\
\hline
\end{tabular}

Abbreviation: BMI, body mass index.

Note: Data are mean or \% unless otherwise specified, (+/- SD [standard deviation]; $\mathrm{N}$, if missing data). 
e352 ECV with One Cesarean McLaren Jr. et al.

Table 2 Success rate among women with ECV attempt

\begin{tabular}{|l|l|l|l|}
\hline Characteristic & No history of cesarean delivery & History of Cesarean delivery & $p$-Value \\
\hline$n$ & 9,976 & 715 & \\
\hline ECV success rate & 86.4 & 80.6 & $<0.001$ \\
\hline${ }^{\mathrm{T}}$ Fetal presentation at birth & & & \\
\hline Vertex & $97.2(8,523)$ & $94.4(567)$ & $<0.001$ \\
\hline Breech & $1.3(8,523)$ & $3.5(567)$ & $<0.001$ \\
\hline Other & $1.4(8,523)$ & $2.1(567)$ & 0.181 \\
\hline
\end{tabular}

Abbreviations: ECV, external cephalic version; TOLAC, trial of labor after cesarean.

${ }^{a}$ Successful ECV; $n=8,616$ and 576, respectively.

Note: Data are \% unless otherwise specified ( $\mathrm{N}$, if missing data).

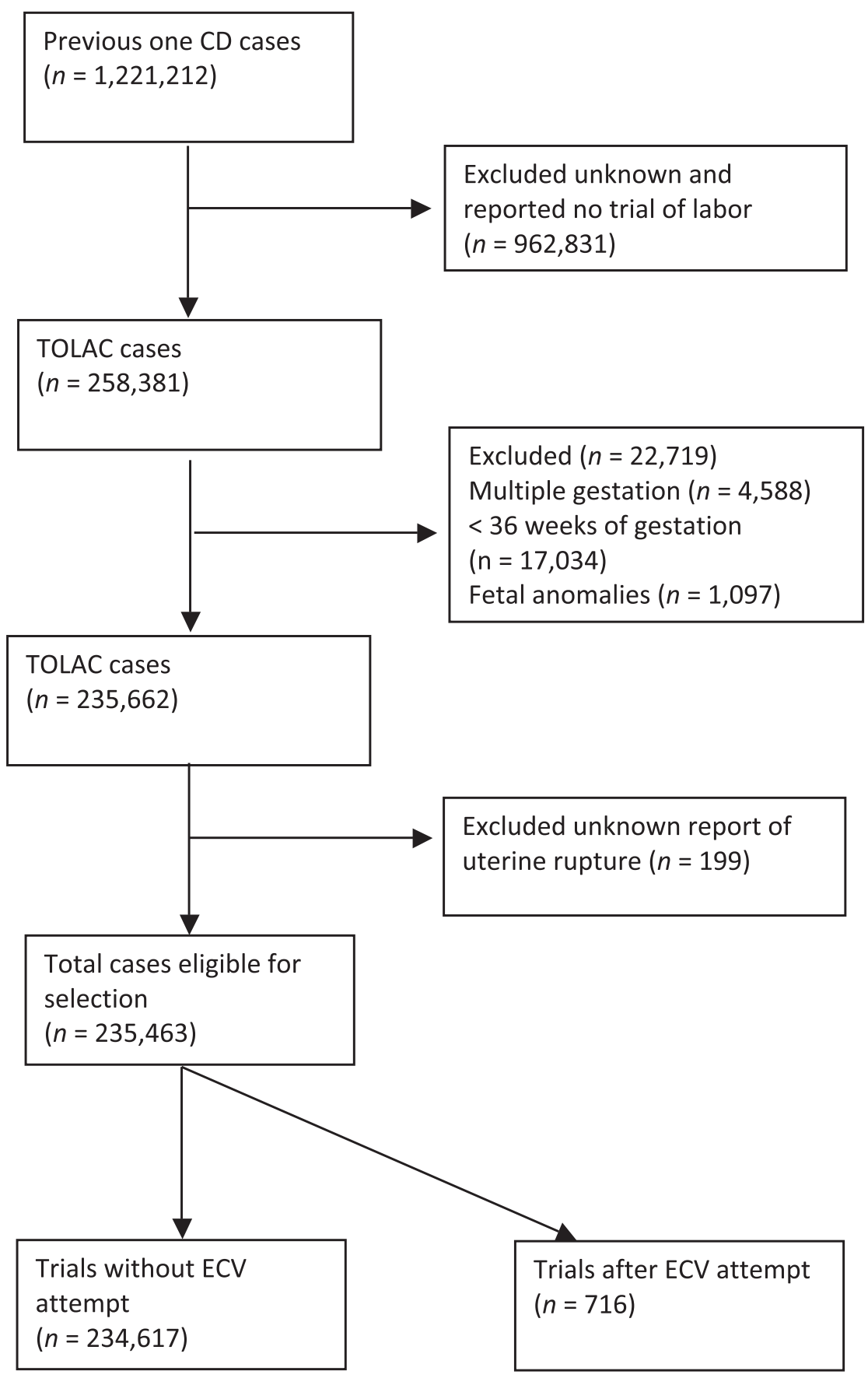

Fig. 2 Cohort selection flow diagram of study 2. CD, cesarean delivery; ECV, external cephalic version; TOLAC, trial of labor after cesarean delivery. 
Table 3 Maternal and obstetrical characteristics among women with one previous cesarean delivery

\begin{tabular}{|l|l|l|l|}
\hline Characteristic & $\begin{array}{l}\text { TOLAC without } \\
\text { ECV attempt }\end{array}$ & $\begin{array}{l}\text { TOLAC after } \\
\text { ECV attempt }\end{array}$ & $p$-Value \\
\hline$n$ & 234,617 & 716 & \\
\hline $\begin{array}{l}\text { Maternal } \\
\text { age (y) }\end{array}$ & $30.3+/-5.2$ & $30.7+/-5.2$ & 0.049 \\
\hline BMI & $27.1+/-6.5$ & $26.8+/-6.2$ & 0.390 \\
\hline $\begin{array}{l}\text { Gestational } \\
\text { age (wk) }\end{array}$ & $39.2+/-1.6$ & $39.3+/-1.6$ & 0.065 \\
\hline $\begin{array}{l}\text { Birth } \\
\text { weight (g) }\end{array}$ & $\begin{array}{l}3,391.9+/ \\
-480.4\end{array}$ & $\begin{array}{l}3,436.6+/ \\
-487.7\end{array}$ & 0.013 \\
\hline
\end{tabular}

Abbreviations: BMI, body mass index; ECV, external cephalic version; TOLAC, trial of labor after cesarean.

Note: Data are mean unless otherwise specified, (+/- SD [standard deviation]).

Table 4 Mode of delivery among TOLAC after ECV attempts

\begin{tabular}{|l|l|l|l|}
\hline Characteristic & $\begin{array}{l}\text { TOLAC without } \\
\text { ECV attempt }\end{array}$ & $\begin{array}{l}\text { TOLAC after } \\
\text { ECV attempt }\end{array}$ & $p$-Value \\
\hline$n$ & 234,617 & 716 & \\
\hline $\begin{array}{l}\text { ECV success } \\
\text { rate }\end{array}$ & - & 80.6 & \\
\hline $\begin{array}{l}\text { a Mode of } \\
\text { delivery }\end{array}$ & & & 0.096 \\
\hline Vaginal & 68.5 & 67.9 & 0.042 \\
\hline Operative & 5.1 & 6.9 & 0.494 \\
\hline Cesarean & 26.4 & 25.1 & \\
\hline
\end{tabular}

Abbreviations: ECV, external cephalic version, TOLAC, trial of labor after cesarean.

${ }^{a}$ Successful ECV; $n=577$.

Note: Data are \% unless otherwise specified.

\section{Discussion}

This study demonstrated that women, with one CD, who underwent a TOLAC with a preceding ECV (ECV), had an increased risk of maternal transfusion, an unplanned hysterectomy, and low Apgar's scores compared with women who underwent TOLAC without a preceding ECV. These results differ from prior investigations.

Flamm et al reported no serious maternal or fetal outcomes among 56 patients with previous CD who underwent ECV. ${ }^{5}$ In addition, de Meeus et al reported only transient vaginal bleeding as a complication among 38 women with previous CD after ECV. ${ }^{7}$ Recently, Weill and Pollack reported no significant complications among 158 patients with and without previous $C D$ in a retrospective study of a prospective cohort of ECV attempts. ${ }^{9}$ These findings may differ from ours due to the low prevalence of adverse maternal and neonatal outcomes and inadequately powered prior studies. We found an increased risk of maternal blood transfusion and an unplanned hysterectomy compared with women without a preceding ECV. A known complication of ECV is placenta abruption which was demonstrated to occur in $0.18 \%$ of ECV in a meta-analysis. ${ }^{4}$ Thus, a possible explanation for the increased risk of maternal blood transfusion and peripartum hysterectomy in women with a preceding ECV may be due to a higher prevalence of abruption in this group. Friedman et al. demonstrated that abruption had an adjusted OR of 2.98 (95\% CI: 2.52-3.2) for peripartum hysterectomy in a population-based study. ${ }^{17}$

The success rate of ECV among multiparous women with previous CD in our study (80.6\%) was slightly higher than the reported pooled rate in the literature $(73.6 \%),{ }^{5-12}$ possibly because all patients in our cohort were multiparous. The success rate may have been smaller in women with a prior scar due to decreased effort in the procedure from providers

Table 5 Risks of maternal and neonatal complications among TOLACs

\begin{tabular}{|c|c|c|c|c|}
\hline & $\begin{array}{l}\text { TOLAC without ECV } \\
\text { attempt }(n=234,617)\end{array}$ & $\begin{array}{l}\text { TOLAC after ECV } \\
\text { attempt }(n=716)\end{array}$ & $\begin{array}{l}\text { Unadjusted OR } \\
(95 \% \mathrm{Cl})\end{array}$ & $\begin{array}{l}\text { adjusted OR } \\
(95 \% \mathrm{Cl})\end{array}$ \\
\hline \multicolumn{5}{|l|}{ Maternal complications } \\
\hline Blood transfusion & $914(0.39)$ & $7(0.98)$ & $2.52(1.01-5.25)$ & $2.48(1.17-5.23)$ \\
\hline Uterine rupture & $532(0.23)$ & - & - & - \\
\hline Hysterectomy & $134(0.06)$ & $3(0.42)$ & $7.36(1.50-22.06)$ & $6.90(2.19-21.78)$ \\
\hline ICU admission & $336(0.14)$ & $1(0.14)$ & $0.98(0.25-5.49)$ & $0.92(0.13-6.57)$ \\
\hline \multicolumn{5}{|l|}{ Neonatal complications } \\
\hline 5-min Apgar's score $<7$ & $3,516(1.5)[233,874]$ & $18(2.5)[714]$ & $1.69(0.10-2.7)$ & $1.76(1.10-2.82)$ \\
\hline Immediate assisted ventilation & $6,944(3.0)[234,399]$ & $28(3.9)$ & $1.33(0.88-1.95)$ & $1.37(0.94-2.01)$ \\
\hline Assisted ventilation for $>6 \mathrm{~h}$ & $1,426(0.6)[234,399]$ & $8(1.1)$ & $1.85(0.79-3.67)$ & $1.97(0.98-3.96)$ \\
\hline Neonatal seizure & $99(0.04)[234,399]$ & - & - & - \\
\hline NICU admission & $12,838(5.5)[234,399]$ & $42(5.9)$ & $1.08(0.77-1.47)$ & $1.16(0.85-1.59)$ \\
\hline
\end{tabular}

Abbreviations: $\mathrm{MBI}$, body mass index; $\mathrm{Cl}$, confidence intervals; $\mathrm{ECV}$, external cephalic version, ICU, intensive care unit; NICU, neonatal intensive care unit; OR, odds ratio; TOLAC, trial of labor after cesarean.

${ }^{a}$ Neonatal outcomes adjusted for maternal BMI, gestational age at delivery and birth weight. Maternal outcomes adjusted for age, maternal BMI, cesarean delivery.

Note: Data are $n(\%)(\mathrm{N}$, if there are missing data), unless otherwise stated. 
because of fear of uterine rupture. Although the success rate among women with a prior CD was significantly smaller than that of multiparous women without a previous $C D$, the mode of delivery was similar between the groups. Thus, we believe the absolute difference is too small to require any modification of standard preprocedure counseling to patients. The rate of vaginal birth, after cesarean, after successful ECV (74.9\%) was similar to a previously reported rate of vaginal birth after cesarean of $79 \%$ among 195 women, after successful ECV. ${ }^{5,9,12}$

The limitations of our study must be acknowledged. The cohorts were derived from large administrative databases which may include data entry errors and miscoding. We attempted to minimize such errors by excluding incomplete records from analyses. Also, we were limited to the variables that were collected in the original dataset. These sorts of datasets do not permit consideration of all potentially interesting confounders. We did adjust for maternal BMI, gestational age at delivery, and birth weight for adverse neonatal outcomes in women undergoing TOLAC with and without preceding ECV attempt. In regard to adverse maternal outcomes, we adjusted for maternal age, maternal BMI, and CD. Further, there is no reason to suspect that there is a systemic bias whereby one group or the other is different in regard to some of the things we could not measure, for example, amniotic fluid index (AFI). The ability to assess a large number of participants acts as a counterweight to this limitation. Also, we were not able to evaluate other known complications of ECV, such as placenta abruption. However, we have surrogates for such complications (e.g., blood transfusion for placental abruption). Finally, we are unable to know the timing of the ECV in relation to the unplanned hysterectomy, thus we cannot evaluate the risk of uterine rupture at time of ECV. However, we found no cases of uterine rupture in women with preceding ECV in this cohort, so the risk of rupture is not more than the risk of rupture during a trial of labor without ECV.

There are strengths to this study. We were able to include large number of external cephalic attempts among women with one previous $\mathrm{CD}(n=716)$ among a contemporaneous obstetrical cohort, allowing us to perform analyzes to evaluate serious adverse outcomes that have low prevalence. We also were not limited to data from a single site which might limit the generalizability of results. In addition, we were able to confirm the efficacy of ECV with one prior cesarean, reported in smaller prior studies, with larger numbers.

In conclusion, we found that the success rate of ECV in multiparous women with one prior $C D$ was significantly smaller than without prior $C D$. However, vaginal birth after cesarean delivery (VBAC) after ECV in women with one prior $C D$ was similar to what is reported in the literature and the absolute difference in success rates seen was small, and thus should not discourage providers from offering ECV to eligible women with one prior $\mathrm{CD}$. We also found an increased risk of maternal blood transfusion, unplanned hysterectomy, and low Apgar's scores among women who underwent TOLAC preceding an ECV attempt. While the absolute risks of these morbidities are low, caution should be undertaken among women undergoing TOLAC after ECV.

\section{References}

1 Hofmeyr GJ, Kulier R, West HM. External cephalic version for breech presentation at term. Cochrane Database Syst Rev 2015; ((04):CD000083

2 Hartnack Tharin JE, Rasmussen S, Krebs L. Consequences of the term breech trial in Denmark. Acta Obstet Gynecol Scand 2011;90 (07):767-771

3 American College of Obstetricians and Gynecologists' Committee on Practice Bulletins-Obstetrics. Practice bulletin no. 161: external cephalic version. Obstet Gynecol 2016;127(02): e54-e61

4 Grootscholten K, Kok M, Oei SG, Mol BW, van der Post JA. External cephalic version-related risks: a meta-analysis. Obstet Gynecol 2008;112(05):1143-1151

5 Flamm BL, Fried MW, Lonky NM, Giles WS. External cephalic version after previous cesarean section. Am J Obstet Gynecol 1991;165(02):370-372

6 Schachter M, Kogan S, Blickstein I. External cephalic version after previous cesarean section-a clinical dilemma. Int J Gynaecol Obstet 1994;45(01):17-20

7 de Meeus JB, Ellia F, Magnin G. External cephalic version after previous cesarean section: a series of 38 cases. Eur J Obstet Gynecol Reprod Biol 1998;81(01):65-68

8 Abenhaim HA, Varin J, Boucher M. External cephalic version among women with a previous cesarean delivery: report on 36 cases and review of the literature. J Perinat Med 2009;37(02):156-160

9 Weill Y, Pollack RN. The efficacy and safety of external cephalic version after a previous caesarean delivery. Aust N Z J Obstet Gynaecol 2017;57(03):323-326

10 Sela HY, Fiegenberg T, Ben-Meir A, Elchalal U, Ezra Y. Safety and efficacy of external cephalic version for women with a previous cesarean delivery. Eur J Obstet Gynecol Reprod Biol 2009; 142(02): 111-114

11 Regalia AL, Curiel P, Natale N, et al. Routine use of external cephalic version in three hospitals. Birth 2000;27(01):19-24

12 Keepanasseril A, Anand K, Soundara Raghavan S. Matched cohort study of external cephalic version in women with previous cesarean delivery. Int J Gynaecol Obstet 2017;138(01):79-83

13 Landon MB, Hauth JC, Leveno KJ, et al; National Institute of Child Health and Human Development Maternal-Fetal Medicine Units Network. Maternal and perinatal outcomes associated with a trial of labor after prior cesarean delivery. N Engl J Med 2004;351(25): 2581-2589

14 Lappen JR, Hackney DN, Bailit JL. Outcomes of term induction in trial of labor after cesarean delivery: analysis of a modern obstetric cohort. Obstet Gynecol 2015;126(01):115-123

15 Rijnders M, Offerhaus P, van Dommelen P, Wiegers T, Buitendijk S. Prevalence, outcome, and women's experiences of external cephalic version in a low-risk population. Birth 2010;37(02):124-133

16 Caukwell S, Joels LA, Kyle PM, Mills MS. Women's attitudes towards management of breech presentation at term. J Obstet Gynaecol 2002;22(05):486-488

17 Friedman AM, Wright JD, Ananth CV, Siddiq Z, D’Alton ME, Bateman BT. Population-based risk for peripartum hysterectomy during low- and moderate-risk delivery hospitalizations. Am J Obstet Gynecol 2016;215(05):640.e1-640.e8 